$>$ Hormis leurs rôles cruciaux dans la présentation antigénique aux lymphocytes $T \mathrm{CD}^{+}$et dans la susceptibilité de l'organisme aux maladies autoimmunes, les molécules du complexe majeur d'histocompatibilité $(\mathrm{CMH})$ de classe II ont la particularité d'être capables de transmettre des signaux à travers la membrane cellulaire. Ces signaux retentissent sur les diverses fonctions biologiques de ces molécules. Paradoxalement, les domaines cytoplasmiques et transmembranaires de ces molécules sont dépourvus des motifs classiques de signalisation. La mise en évidence des molécules du CMH de classe II, dans les microdomaines membranaires appelés radeaux lipidiques, enrichis en kinases et molécules adaptatrices, pourrait contribuer à élucider les mécanismes par lesquels les molécules du CMH de classe II transmettent leurs signaux. <

\section{Complexe majeur d'histocompatibilité de classe II : diversité fonctionnelle}

Marlène Bouillon, Walid M. Mourad

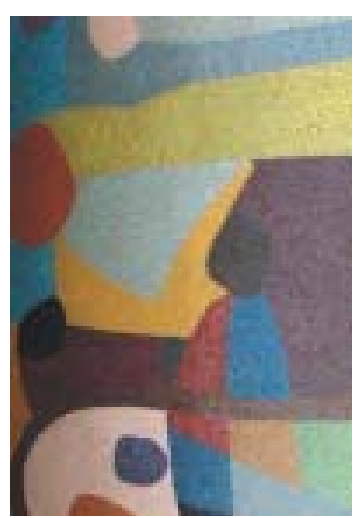

pour DP et DQ, et un ou deux hétérodimères liés pour DR.

Chaque chaîne est composée de deux domaines extracellulaires, d'un domaine transmembranaire et d'une courte queue cytoplasmique (12-18 acides aminés). Le polymorphisme de ces molécules est principalement situé dans le premier domaine de la molécule, $\alpha 1$ et $\beta 1$, et regroupé en 2 à 4 régions hypervariables [1]. Cette variation structurale représente la base fondamentale permettant la spécificité antigénique. Le peptide antigénique de 13 à 18 acides aminés, fruit de l'endocytose et de la dégradation d'un antigène exogène, se fixe dans la niche peptidique située à l'extrémité des domaines $\alpha$ l et $\beta$ l où réside la majorité des acides aminés hautement polymorphiques. L'expression constitutive de ces molécules est restreinte aux cellules présentatrices d'antigène (CPA), mais elle peut être induite sur des lymphocytes T activés et des cellules non immunes. 
Outre leur fonction de présentation antigénique, les molécules du CMH de classe II jouent un rôle important dans la susceptibilité aux maladies auto-immunes telles que l'arthrite rhumatoïde, le diabète de type I et la sclérose multiple [2]. II semble que les régions polymorphiques de certains allèles de prédisposition, particulièrement des familles HLA-DR et HLA-DQ, présentent des auto-antigènes qui activent une population de lymphocytes T potentiellement autoréactifs.

\section{Les molécules du CMH de classe II sont les récepteurs spécifiques des superantigènes}

Mis à part leur rôle dans l'interaction avec le CD4 et le LAG-3 (gène d'activation du lymphocyte-3), les molécules du CMH de classe II agissent comme récepteurs des superantigènes (SAg) [3]. Ces SAg peuvent être d'origine bactérienne ou virale; ils se lient aux molécules du CMH de classe II sans dégradation préalable. Bien que la majorité des SAg se lient aux régions non polymorphiques des molécules du CMH de classe II, la liaison de certains SAg tels que la toxine du du syndrome de choc toxique (TSST-1) et le mitogène de Mycoplasma arthritiditis (MAM) est affectée par la nature du peptide antigénique présent dans la niche peptidique [4, 5]. Les SAg liés aux molécules du CMH de classe II sont reconnus par la région variable (V $\beta$ ) des récepteurs des lymphocytes $T$ (RcT), permettant l'activation polyclonale de ces derniers [3]. En outre, du fait de leur mode d'interaction avec les molécules du CMH de classe II, certains SAg favorisent leur dimérisation ou leur oligomérisation [6].

\section{Fonctions biologiques des molécules du CMH de classe II}

La reconnaissance du complexe peptide antigénique/CMH de classe II par le RcT/CD4 induit l'activation des lymphocytes T et des CPA [7]. Devant le constat suggérant une fonction jusque-là inconnue pour les molécules du CMH de classe II, des anticorps spécifiques et des SAg ont été utilisés pour mimer l'action du RcT/CD4. II a été démontré que l'engagement des molécules du CMH de classe II induit l'adhérence hétéro- et homotypique, la prolifération et la différenciation des lymphocytes $B$, la production de cytokines pro-inflammatoires et de médiateurs de l'inflammation, l'expression de molécules co-stimulatrices et, dans certaines circonstances, la mort cellulaire [8]. De plus, l'utilisation de ligands monovalents et bivalents a permis de démontrer l'importance de la dimérisation des molécules du CMH de classe II dans la plupart de ces événements [9].

\section{Bases structurales et biochimiques de la signalisation par les molécules du CMH de classe II}

La liaison des molécules CMH de classe II entraîne l'activation de deux voies de signalisation distinctes: I'une nécessitant les résidus 225,227 et 228 présents dans le domaine cytoplasmique de la chaîne $\beta$, et l'autre dépendante du domaine transmembranaire de la chaîne $\beta[10,11]$. Les deux voies sont toutefois partiellement coopératives puisque l'inactivation de l'une ou l'autre empêche les molécules du CMH de classe II d'induire la différenciation des cellules $B$ [10].

Dans les cellules B quiescentes de souris, l'engagement des molécules du CMH de classe II entraîne l'élévation d'AMPc avec pour effet la translocation nucléaire de la protéine kinase $C \beta$ (PKC $\beta$ ) [12]. Le traitement de ces cellules avec des analogues de I'AMPc conduit à la mort cellulaire tout comme l'engagement des molécules du CMH de classe II [13]. Dans les cellules activées, il y a activitation de Lyn, une protéine tyrosine kinase (PTK) de la famille src (src-PTK), et de Syk, une PTK non-Src. Ces PTK phosphorylent la phospholipase $C \gamma(P L C \gamma)$, permettant la production d'inositol 1,4,5-triphosphate $\left(\mathrm{IP}_{3}\right)$ et de diacylglycérol (DAG), qui sont respectivement responsables de la mobilisation du $\mathrm{Ca}^{2+}$ et de l'activation de la PKC $\beta[8,12]$. Dans les lymphocytes B humains (Figure 1), la mobilisation des molécules du CMH de classe II par des anticorps monoclonaux (AcM) ou des SAg induit l'activation rapide de src-PTKs, particulièrement Fgr, Hck et Lyn, sans activation préalable des cellules [12]. Les PTK phosphorylent les PLC $\gamma 1$ et 2, conduisant à la production de DAG et d'IP ${ }_{3}$. Le $D A G$ active la translocation de la PKC ( $\alpha$ et $\beta$ II) vers la membrane plasmique tandis que I'IP ${ }_{3}$ permet la mobilisation du $\mathrm{Ca}^{2+}$ intracellulaire [11]. Fait intéressant, seules les cellules $B$ humaines activées sont susceptibles à l'apoptose [14], suggérant que l'engagement des molécules du CMH de classe II permet de maintenir l'homéostasie de la réponse immunitaire. En comparant le modèle souris et le modèle humain, on constate que le stade d'activation des cellules $B$ est l'élément déterminant dans l'induction de la mort cellulaire car l'engagement des molécules du CMH de classe II entraîne finalement l'activation de la PKC dans les deux modèles via des seconds messagers différents. 
L'activation via les molécules du CMH de classe II de la voie des MAPK (mitogen-activated protein kinases) et des PTK a également été mise en évidence dans les monocytes humains isolés du sang périphérique [15] et les lignées monocytaires [16]. Dans les monocytes, l'activation de p38 stimule la production d'IL-1 $\beta$ et d'IL-10 alors que l'activation de la voie impliquant ERKI/2 a peu d'effet sur la production d'IL-1 $\beta$ mais a un effet négatif sur la production d'IL-10. L'activation des PTK et de la PKC joue un rôle essentiel dans l'adhérence et la production de cytokines médiées par les molécules du CMH de classe II [17]. La stimulation par les SAg induit également l'activation de la phospholipase $A_{2}\left(P L A_{2}\right)$ et de la cyclooxygénase-2 (COX-2) [18].

Le couplage des molécules du CMH de classe II à ces diverses voies de signalisation demeure néanmoins un mystère. $\varepsilon n$ effet, le motif du domaine cytoplasmique de la chaîne $\beta$ ne correspond pas à celui retrouvé habituellement dans les récepteurs couplés aux protéines $G$. De plus, l'activation des PTK implique des interactions avec d'autres protéines transmembranaires pour faire le lien avec les PTK cytoplasmiques.

\section{Les radeaux lipidiques et la signalisation}

Une étape importante a été récemment franchie lors de l'identification et de la caractérisation de microdomaines dans la membrane plasmique des cellules eucaryotes. Ces microdomaines plus connus sous l'appellation de radeaux lipidiques (raft), sont constitués principalement de cholestérol et de sphingolipides (Figure 2A). Les radeaux lipidiques sont insolubles dans les détergents non-ioniques et peuvent être isolés des autres constituants par ultracentrifugation en gradient de sucrose [19]. L'utilisation d'agents ayant la capacité d'extraire (méthyl$\beta$-cyclodextrine $[M \beta C D]$ ) ou de séquestrer (filipine, nystatine) le cholestérol a contribué à démontrer l'importance de ces microdomaines. Toutefois, ce sont les approches utilisant des cellules intactes (microscopie confocale, fluorescence resonance energy transfert microscopy, single-particle tracking) qui ont permis de confirmer l'existence de ces microdomaines, leur composition, leur taille $(50-70 \mathrm{~nm})$ et leur distribution [20]. On admet maintenant que ces structures de petite taille, après une stimulation, peuvent fusionner pour former un macro-radeau, rapprochant et concentrant ainsi diverses molécules.

K. Simons et D. Toomre [19] ont dressé une liste assez

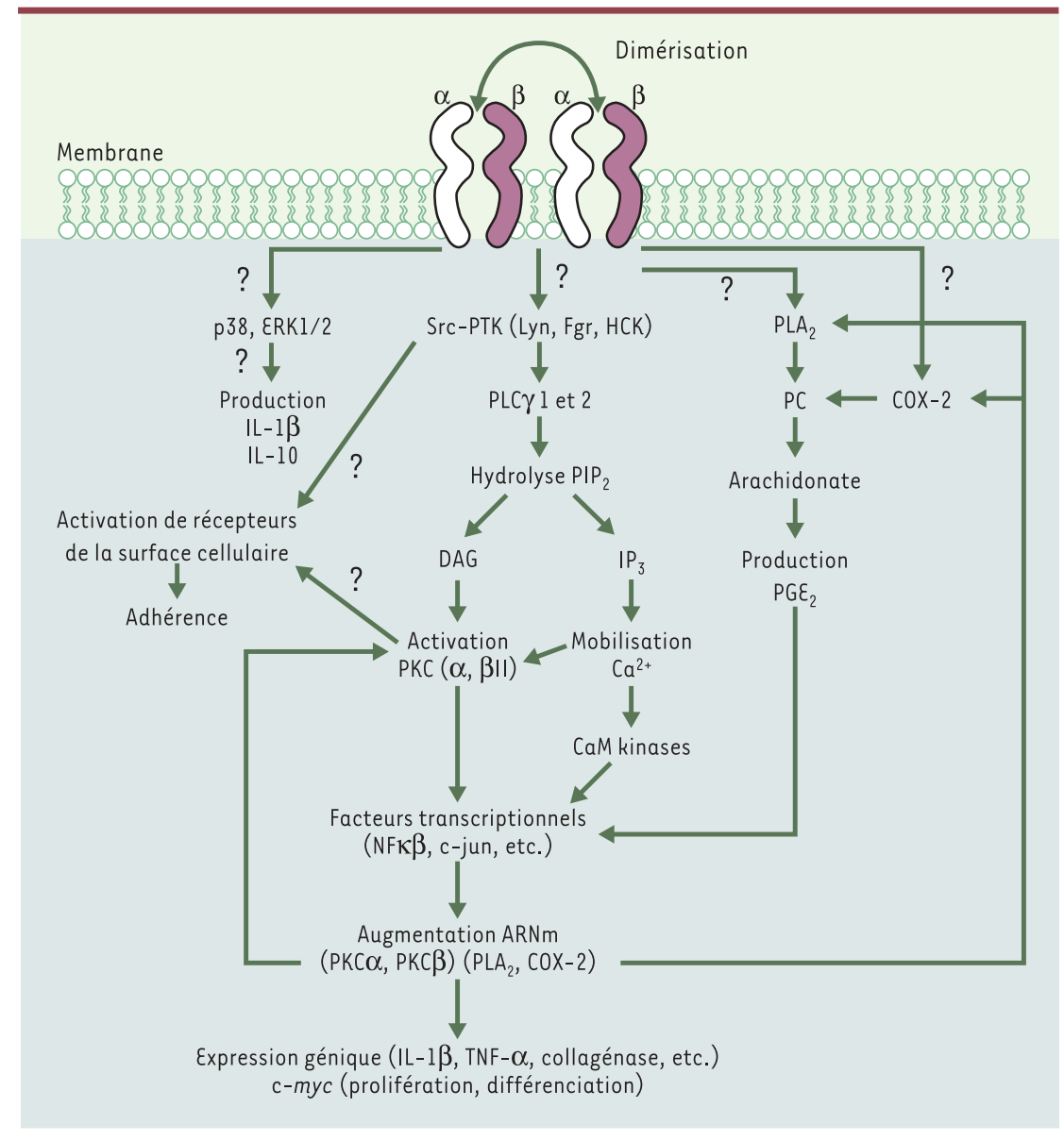

Figure 1. Événements biochimiques associés à la signalisation induite via les molécules du complexe majeur d'histocompatibilité (CMH) de classe II. La dimérisation des molécules du CMH de classe II entraîne l'activation de diverses cascades de signalisation. Ainsi, l'activation de protéine tyrosine kinases (PTK), particulièrement les src-PTK, est à l'origine de la production de seconds messagers (diacylglycérol [DAG], inositol 1, 4, 5-triphosphate [IP3], $\mathrm{Ca}^{2+}$ ) lesquels activent la protéine kinase C (PKC). L'activation des PTK et de la PKC favorise l'adhérence hétéroet homotypique mais également l'augmentation de la transcription de plusieurs gènes, dont ceux de la phospholipase $A_{2}$ (PLA2) et de la cyclo-oxygénase-2 (COX-2). Ces dernières sont impliquées dans la production de médiateurs inflammatoires. De plus, l'activation des PTK et de la PKC permet la transcription de gènes impliqués dans la prolifération et la différenciation des lymphocytes $B$, et de gènes responsables de la production de cytokines pro-inflammatoires. Parallèlement, l'activation de la voie des MAPK (mitogen activated protein kinase) p38 et ERK1/2 se traduit par la production d'IL-1 $\beta$ et d'IL-10. CaM: calmoduline; $P G \varepsilon_{2}$ : prostaglandine $\varepsilon_{2} ;$ PLC $\gamma$ : phospholipase $\mathrm{C} \gamma$; PIP : phosphatidylinositiol diphosphate ; PC : phosphatidylcholine. 
exhaustive des protéines incluses ou exclues des radeaux lipidiques. Ainsi, la présence de protéines ancrées par un glycosyl-phosphatidylinositol (GPI), la présence de Src-PTK doublement acylées (particulièrement Lyn), de sous-unités $\alpha$ des protéines $G, H$-Ras, la présence de protéines membranaires et transmembranaires palmitoylées, et celles de nombreux récepteurs ( $F c \varepsilon R I, R c T, R c B$ ) ont été établies alors que certaines protéines sont spécifiquement exclues (CD22 et CD45 dans les lymphocytes B). Ces microdomaines concen-

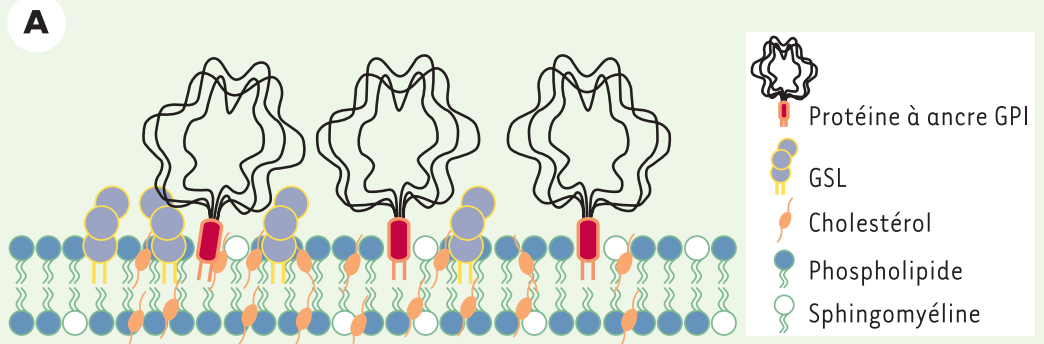

B

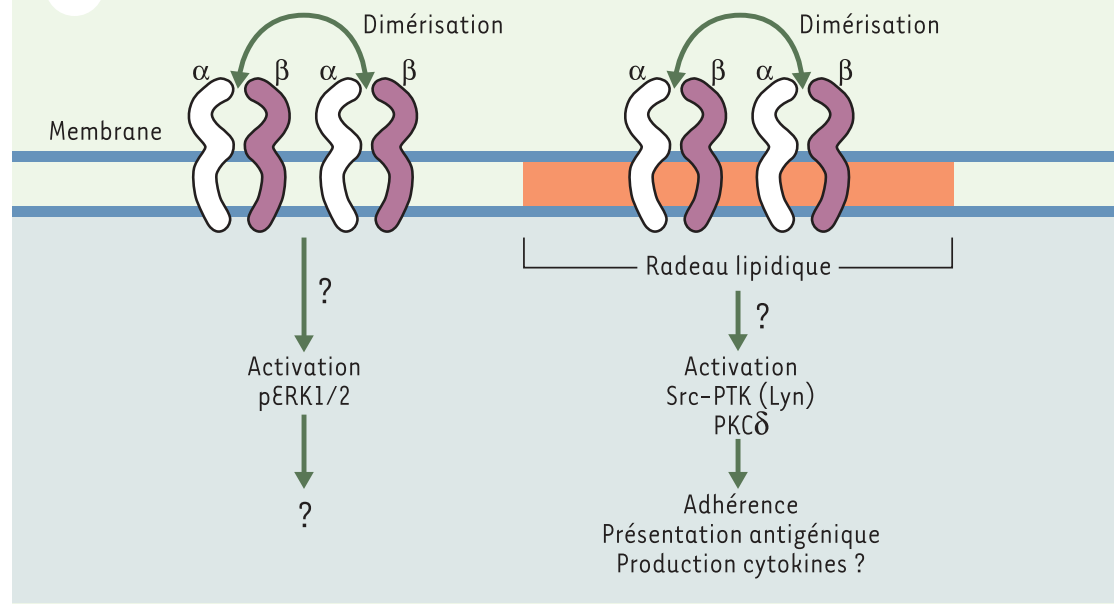

Figure 2. Contribution des radeaux lipidiques à la signalisation relayée par les molécules du CMH de classe II. A. Le cholestérol s'intercale dans la double couche lipidique formée par la sphingomyéline, les glycosphingolipides (GSL), et les phospholipides, créant ainsi une zone lipidique ordonnée dans la membrane plasmique. On retrouve également des protéines ancrées par un glycosylphosphatidylinositol (GPI). B. La dimérisation de molécules du CMH de classe II par un ligand bivalent permet l'activation de deux voies de signalisation distinctes selon la localisation des molécules HLA-DR. Ainsi, l'activation de la voie impliquant ERK1/2 met en jeu des molécules qui se retrouvent à l'extérieur des radeaux lipidiques tandis que l'activation des src-PTK, notamment Lyn, est dépendante de la localisation des molécules HLA-DR dans ces microdomaines. Toutefois, le recrutement de molécules additionnelles dans ce compartiment n'est pas nécessaire à l'activation des src-PTK. L'adhérence, la présentation antigénique et la production de cytokines pro-inflammatoires sont des événements qui sont relayés par des molécules de classe II présentes dans les radeaux lipidiques. PKC $\delta$ : protéine kinase $C \delta$. trent, séquestrent ou encore excluent certaines pro; en outre, ils restreignent la diffusion latérale la signalisation relayée par le récepteur des lymphocytes $T$ (RcT) [21] et le récepteur des lymphocyte B (RcB) [22] soit bien établi, leur implication dans la signalisation induite via les molées du CMH de classe II commence à peine à faire l'objet d'études. travaux récents ont démontré la présence constitutive des molécules radeaux lipidiques de lignées cellulaires $B$ (humaines et murines) [23] et de cellules dendritiques [24]. Ainsi, H.A. Anderson et al. [23] ont établi que $50 \%$ des molécules de classe II présentes à la surface cellulaire étaient associées aux radeaux lipidiques. En outre, la concentration des molécules de classe II dans ce compartiment semblait faciliter la présentation antigénique lorsque les concentrations de l'antigène étaient restreintes. Fait intéressant, dans la lignée cellulaire humaine B Raji, la liaison des molécules du CMH de classe II par un anticorps spécifique entraine la co-localisation de la $\operatorname{PKC} \delta$ et de ces dernières dans les radeaux lipidiques [25]. Cette observation soulève néanmoins une question importante: quels sont les mécanismes responsables du couplage des molécules de classe II aux voies de signalisation? Dans les cellules $B$ humaines, il a été démontré que les molécules du CMH de classe II, particulièrement les HLA-DR, s'associent avec plusieurs récepteurs de la surface cellulaire tels que le CD19/CD21, CD20, CD40 [26, 27 , et les membres de la famille des tétraspans (CD9, CD37, CD53, CD81, CD82) $[28,29]$. Or, après oligomérisation, certaines de ces molécules se retrouvent dans les radeaux lipidiques. P. Lang et al. [30] ont même démontré que la stimulation antigénique de cellules $B$ quiescentes de souris induisait l'association des molécules du CMH de classe II avec des hétérodimères (CD79a/CD79b) et que la phosphorylation de ces derniers permettait l'activation de PTK. La présence de ces hétérodimères dans les radeaux lipidiques de cellules $B$ a d'ailleurs été démontrée lors de la translocation du BcR [31].

Concernant les monocytes, l'équipe de R.D. Huby [32], en utilisant la lignée 
THP-1 stimulée par l'interféron- $\gamma$ (INF- $\gamma$ ), a démontré que seule l'oligomérisation avait pour conséquence la translocation des molécules HLA-DR dans les radeaux lipidiques. De plus, le recrutement des molécules de classe II dans les microdomaines est associé au début de l'activation des PTK, notamment Lyn, comme le démontre l'abolition de cette activation à la suite de l'utilisation de $M \beta C D$ [32]. En revanche, nos travaux ont permis de démontrer la présence constitutive des molécules HLA-DR dans les radeaux lipidiques des cellules THP- 1 stimulées par l'INF- $\gamma$ ou transfectées avec le transactivateur CIITA [16]. La dimérisation des molécules HLA-DR par des anticorps est suffisante pour induire l'activation des PTK et des MAPK (mitogen activated protein kinase) $\varepsilon R K 1 / 2$ (Figure $2 B$ ). L'activation des PTK, particulièrement Lyn, est dépendante de la localisation des molécules HLA-DR dans les microdomaines mais ne requiert pas le recrutement additionnel de molécules HLA-DR dans ce compartiment. L'activation des PTK, particulièrement les src-PTK, est impliquée dans l'adhésion homotypique. L'activation de la voie mobilisant $\varepsilon R K 1 / 2$ est, quant à elle, indépendante de la localisation des molécules HLA-DR dans les radeaux lipidiques. Enfin, les domaines cytoplasmiques ne sont pas nécessaires à la localisation des HLA-DR dans les radeaux lipidiques.

Là encore, ces observations posent la question du lien existant entre les molécules du CMH de classe II et les PTK ou les MAPK dans les monocytes puisque ces cellules sont dépourvues des diverses molécules mentionnées ci-dessus pour les cellules B. R.D. Huby et al. [32] proposent que l'agrégation de radeaux lipidiques individuels concentre et stabilise les molécules du CMH de classe II. II y aurait alors activation de src-PTK localisées dans les radeaux lipidiques. Cette activation amorce la phosphorylation de substrats présents dans le compartiment soluble qui font partie d'une grande cascade d'activation de PTK. Le mystère demeure entier quant aux partenaires potentiels impliqués.

\section{Conclusions}

Les radeaux lipidiques facilitent la signalisation de divers récepteurs immuns dans les CPA en leur procurant un environnement riche en kinases, en molécules adaptatrices, et en co-récepteurs, et en les isolant d'éléments de régulation négative comme les phosphatases. De plus, en concentrant ces diverses molécules, ils favorisent la formation d'un synapse immunologique permettant une activation plus efficace de la cellule $T$. $\varepsilon n$ revanche, on n'explique pas comment les molécules de classe II se retrouvent dans ce compartiment. En

effet, elles ne possèdent pas les caractéristiques structurales habituelles (ancre GPI, palmitoylation) des autres molécules dont l'association a été rapportée avec les radeaux lipidiques. De plus, le couplage aux PTK cytoplasmiques n'est pas clair, puisque l'activation de cette voie de signalisation requiert le domaine transmembranaire de la chaîne $\beta$. Des études utilisant des mutants des divers domaines devraient permettre l'identification de résidus importants pour la localisation des molécules de classe II du CMH. Finalement, il faudra déterminer si les molécules de classe II activent directement ou indirectement les PTK et dans ce cas identifier les partenaires en cause. $\diamond$

\section{SUMMARY}

Major histocompatibility complex (MHC) class II : are lipid rafts the missing link?

Aside from their crucial roles in the presentation of nominal antigen to $C D 4^{+} \mathrm{T}$ cells and susceptibility to autoimmune diseases, substantial evidences suggest that MHC class II molecules act as signal tranducer receptors as well. The signals transmitted affect diverse biological functions. Paradoxically, the cytoplasmic and transmembrane domains of these molecules are devoid of classic signaling motifs. The recent discovery of the presence of membrane microdomains, also called lipid rafts, that are enriched in kinases and adaptor molecules, may contribute to the elucidation of the mechanisms by which MHC class II molecules transmit their signals. $\diamond$

\section{RÉFÉRENCES}

1. McDevitt H. Evolution of MHC class II allelic diversity. Immunol Rev 1995 ; 143:113-22.

2. Winchester R. The molecular basis of susceptibility to rheumatoid arthritis. Adv Immunol 1994 ; 56 : 389466.

3. Lavoie P, Thibodeau J, Erard F, Sekaly RP. Understanding the mechanism of action of bacterial superantigens from a decade of research. Immunol Rev 1999 ; 168 : 257-69.
4. Kim J, Urban RG, Strominger J, Wiley DC. Toxic shock syndrome toxin-1 complexed with a class II major histocompatibility molecule HLA-DR1. Science 1994 ; 266 : 1870-4.

5. Etongue-Mayer $P$, Langlois M, Ouellette M, Mourad W. Involvement of zinc in the binding of Mycoplasma arthritidis-derived mitogen to the proximity of the HLA-DR binding groove regardless of histidine 81 of the beta chain. Eur Immunol $2002 ; 32$ : 50-8. 
6. Al Daccak R, Mehindate K, Damdoumi F, et al. Staphylococcal enterotoxin $D$ is a promiscuous superantigen offering multiple modes of interactions with the $\mathrm{MHC}$ class II receptors. J Immunol 1998 ; 160 : 22532.

7. Corley RB, LoCascio NJ, Ovnic M, Haughton G. Two separate functions of class II (la) molecules: T-cell stimulation and $B$-cell excitation. Proc Natl Acad Sci USA 1985 ; 82 : 516-20.

8. Aoudjit F, Al-Daccak R, Léveillé $C$, Mourad W. Effects and mechanisms underlying the interaction of bacterial superantigens with MHC class II-positive cells. In : Thibodeau J, Sékaly R, eds. Bacterial superantigens: Stucture, function and therapeutic potential. New York: Springer-Verlag, 1995 : 147-60.

9. Mehindate K, Thibodeau J, Dohlsten M, Kalland T, Sekaly RP, Mourad W. Cross-linking of major histocompatibility complex class II molecules by staphylococcal enterotoxin A superantigen is a requirement for inflammatory cytokine gene expression. J Exp Med 1995 ; 182 : 1573-7.

10. Harton JA, Van Hagen AE, Bishop GA. The cytoplasmic and transmembrane domains of MHC class II beta chains deliver distinct signals required for $\mathrm{MHC}$ class II-mediated B cell activation. Immunity 1995 ; 3: 349-58.

11. Andre P, Cambier JC, Wade TK, Raetz T, Wade WF. Distinct structural compartmentalization of the signal transducing functions of major histocompatibility complex class II (la) molecules. J Exp Med 1994 ; 179 : 763-8.
12. Watts TH. Signalling via MHC molecules. In : Harnete MM, Rigley KP, eds. Lymphocyte signalling : mechanisms, subversion and manipulation. New york : John Wiley and Sons Ltd, 1997 : 141-61.

13. Newell MK, VanderWall J, Beard KS, Freed JH. Ligation of major histocompatibility complex class II molecules mediates apoptotic cell death in resting B lymphocytes. Proc Natl Acad Sci USA 1993 ; $90: 10459-63$.

14. Truman JP, Ericson ML, Choqueux Seebold CJ, Charron DJ, Mooney NA. Lymphocyte programmed cell death is mediated via HLA class II DR. Int Immunol $1994 ; 6$ : 887-96.

15. Matsuoka T, Tabata H, Matsushita S. Monocytes are differentially activated through HLA-DR, -DQ, and -DP molecules via mitogen-activated protein kinases. J Immunol 2001 ; $166: 2202-8$.

16. Bouillon M, El Fakhry $Y$, Girouard J, Khalil H, Thibodeau J, Mourad W. Lipid raft-dependent and -independent signaling through HLA-DR molecules. J Biol Chem 2003 ; 278 : 7099-107.

17. Kansas GS, Tedder TF. Transmembrane signals generated through $\mathrm{MHC}$ class II, CD19, CD20, CD39, and CD40 antigens induce LFA-1-dependent and -independent adhesion in human $B$ cells through a tyrosine kinase-dependent pathway. J Immunol 1991; 147 : 4094-102.

18. Mehindate K, Al-Daccak R, Dayer JM et al. Superantigen-induced collagenase gene expression in human IFN- $\boldsymbol{\gamma}$ treated fibroblast-like synoviocytes involves prostaglandin $\varepsilon 2$. Evidence for a role of cyclooxygenase- 2 and cytosolic phospholipase A2. J Immunol 1995 ; 155 : 3570-7.
19. Simons K, Toomre D. Lipid rafts and signal transduction. Nat Rev Mol Cell Biol 2000 ; 1 : 31-9.

20. Varma R, Mayor S. GPIanchored proteins are organized in submicron domains at the cell surface. Nature 1998 ; 394 : 798-801.

21. Montixi C, Langlet $C$, Bernard AM, et al. Engagement of T cell receptor triggers its recruitment to low-density detergent-insoluble membrane domains. Embo J 1998 ; 17 : 5334-48.

22. Cheng, PC, Dykstra ML, Mitchell RN, Pierce SK. A role for lipid rafts in B cell antigen receptor signaling and antigen targeting. $)$ Exp Med 1999 ; 190 : 154960.

23. Anderson $H A$, Hiltbold $\varepsilon M$, Roche PA. Concentration of MHC class II molecules in lipid rafts facilitates antigen presentation. Nat Immunol 2000; 1 : 156-62.

24. Kropshofer $\mathrm{H}$, Spindeldreher S, Rohn TA, et al. Tetraspan microdomains distinct from lipid rafts enrich select peptide- MHC class II complexes. Nat Immunol $2002 ; 3: 61-8$.

25. Setterblad N, Becart $S$, Charron D, Mooney N. Signalling via MHC class II molecules modifies the composition of GEMs in APC. Scand I Immunol $2001 ; 54: 87-92$.

26. Léveillé $C$, Chandad $F, A I$ Daccak R, Mourad W. CD40 associates with the MHC class II molecules on human B cells. EurJ Immunol 1999 ; 29 : 351626.

27. Léveillé C, Al Daccak R., Mourad W. CD20 is physically and functionally coupled to MHC class II and CD40 on human B cell lines. Eur J Immunol 1999 ; 29 : 65-74.
28. Bradbury $L \varepsilon$, Kansas GS, Levy S, Evans RL, Tedder TF. The CD19/CD21 signal transducing complex of human B lymphocytes includes the target of antiproliferative antibody1 and Leu- 13 molecules. J Immunol 1992 ; 149 : 284150.

29. Schick MR, Levy S. The TAPA-1 molecule is associated on the surface of $B$ cells with HLA-DR molecules. J Immunol 1993 ; $151: 4090-7$.

30. Lang P, Stolpa JC, Freiberg $B A$, et al. TCR-induced transmembrane signaling by peptide/MHC class II via associated Ig- $\alpha / \beta$ dimers. Science 2001 ; 291 : $1537-$ 40.

31. Dykstra ML, Longnecker R, Pierce SK. Epstein-Barr virus coopts lipid rafts to block the signaling and antigen transport functions of the BCR. Immunity 2001 ; 14 : 57-67.

32. Huby RD, Dearman RJ, Kimber I. Intracellular phosphotyrosine induction by major histocompatibility complex class II requires co-aggregation with membrane rafts. J Biol Chem 1999 ; 274 : 22591-6.
TIRÉS À PART

M. Bouillon 